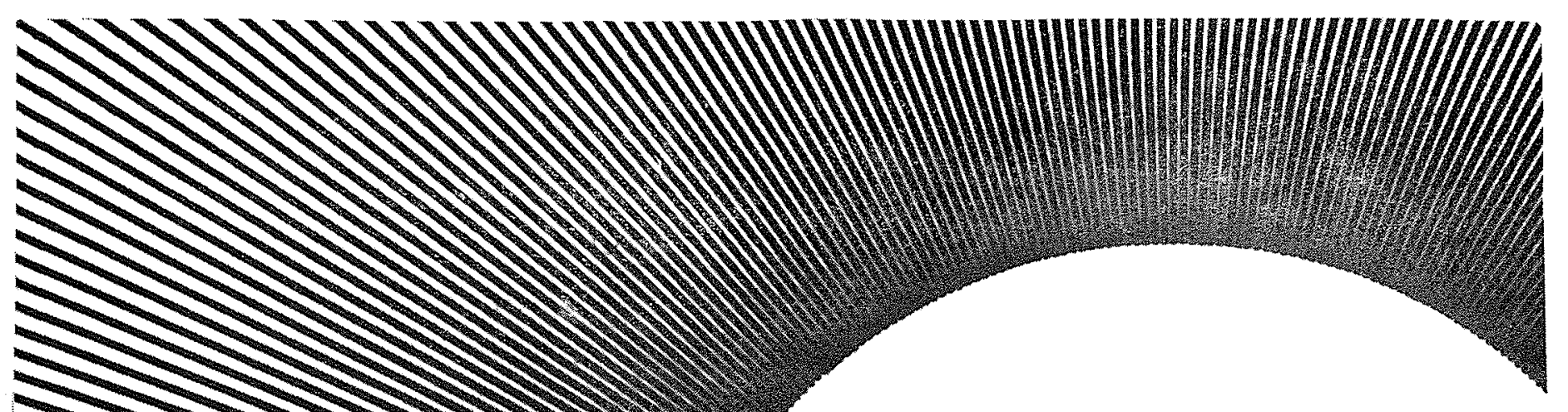

\title{
EXEMPLES DE RÉGÉNÉRATION DE LACS EN SUEDE
}

\author{
par S. BJÖRK \\ Professeur à l'Institut de Limnologie \\ Université de Lund (Suède)
}

Au cours des recherches effectuées à l'Institut de Limnologie de Lund, dans le domaine de cette partie de la défense active de la nature appelée régénération des lacs, les travaux ont été concentrés sur trois types de lacs pollués.

En même temps, se pose le problème de trois types différents de régénération ou de rénovation de lacs. Tous ces exemples impliquent lintervention d'une équipe technicoécologique.

Le premier exemple, illustré par le lac Trummen, dans le Växjö (partie centre-sud de la Suède) est celui d'un lac peu profond, dont les possibilités d'absorption d'eaux usées ont été dépassées, et qui ne s'est pas assaini après la cessation du déversement de celles-ci. Les petits lacs de cette catégorie peuvent être traités en évacuant par pompage les sédiments accumulés (coopération technique avec la Société Skanska Cementgjureriet, division dragages, Stockholm).

Le deuxième exemple est celui du lac plus ou moins profond, dont la masse liquide présente une déficience d'oxygénation dans ses parties inférieures, avec les conséquences limnologiques que cela entraîne. A l'aide d'un apport d'air, on améliore l'oxygénation des couches aquatiques inférieures.

Les expérimentations dans ce domaine sont poursuivies dans le lac Järla à Stockholm et dans le lac Växjö. Ce projet implique un programme de recherche en commun du laboratoire central de la Société suédoise Atlas Copco et de l'Institut de Limnologie.

Le troisième type — dont le lac Hornborga, dans le
Västergötland entre les grands lacs Vänern et Vätern est un exemple - est le lac peu profond dont le niveau a été abaissé de façon considérable.

Afin de régénérer ce lac, encombré đ'une végétation luxuriante en surface, il est nécessaire de remonter le niveau de l'eau.

Avant de le faire, il est toutefois nécessaire de mettre en œuvre des dispositions préparatoires d'ordre limnologique.

Dans tous les cas, l'étude est effectuée en travail d'équipe et les recherches comportent des études comparatives des systèmes écologiques avant, pendant et après l'intervention.

\section{Lac Trummen}

La régénération du lac Trummen a été entreprise en juillet 1970 pour être terminée à l'automne de 1971. Le lac a une superficie de $1 \mathrm{~km}^{2}$ et sa profondeur maximale est de $2 \mathrm{~m}$.

Jusqu'en 1958 le lac Trummen a reçu des eaux usées provenant d'une part des habitations et d'un hôpital, et d'autre part - pendant une plus courte période - d'une usine de transformation de lin.

A l'origine, c'était un lac oligotrophe, courant dans cette région; mais lorsqu'en 1959 l'apport des eaux usées cessa, le lac présentait toutes les caractéristiques d'un lac peu profond dont les capacités d'épuration ont été dépassées : forte expansion de la végétation de surface, efflorescence continue de l'eau, déficience de l'oxygénation et forte mortalité des poissons durant l'hiver. 


\section{S. BJÖRK}

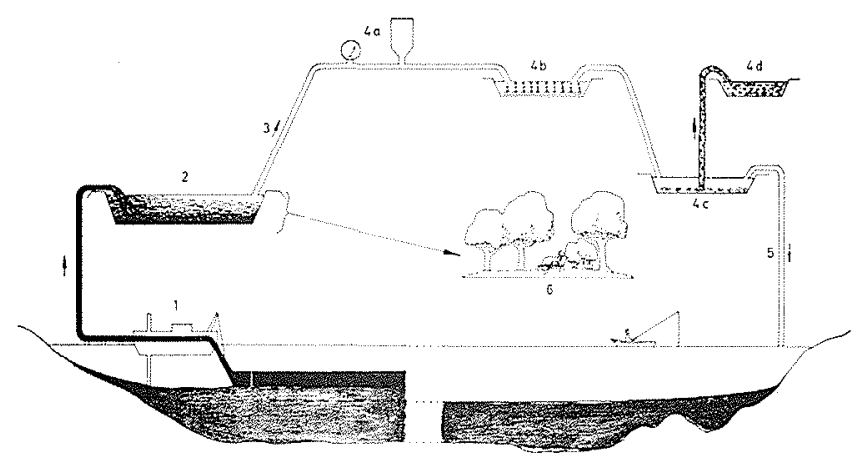

1/Schéma mis en ouvre en vue de l'assainissement du lac Trummen :

1. Drague suceuse / 2. Bassin de décantation / 3. Eau de trop-plein/4. Dispositif de précipitation au sulfate d'alumine $(4 \mathrm{a}$. Dosage automatique; $4 b$. Aération; $4 c$. Décantation; $4 d$. Bas$\sin$ des boues) /5. Eau clarifiée / 6. La zone de décantation actuelle sera sans doute aménagée en terrains de récréation et de loisirs.

Lake Trummen, the practiced scheme for the restoration:

1. Suction dredger / 2. Settling pond / 3. Runoff water / 4. Precipitation with aluminium sulphate ( $4 \mathrm{a}$. automatic dosage; $4 \mathrm{~b}$. aeration; $4 \mathrm{c}$. sedimentation; $4 \mathrm{~d}$. sludge pond) $/ 5$. Clarified run-off water 16 . The area now utilized for sediment aeposition will probably become recreation grounds.

Depuis la cessation du déversement des eaux usées la situation ne s'est pas améliorée; la transparence d'été a été d'environ $20 \mathrm{~cm}$ durant toutes les années 60 .

Dans un proche avenir le lac sera ceinturé de constructions. Dans le plan d'aménagement de la région, la ville de Växjö s'est prononcée en faveur de la régénération du lac afin d'en faire un élément positif dans le problème d'environnement posé par la création d'une zone à haute densité d'habitation.

Le lac Trummen a fait l'objet d'études limnologiques dès 1920 , de sorte que les particularités principales de son évolution étaient connues. A partir de ces études de base les grandes lignes d'un projet de régénération furent publiées en 1966. L'intervention devrait comporter en premier lieu un nettoyage du lac par pompage des sédiments accumulés pendant la période où le lac a reçu des eaux usées.

Afin d'étudier sous tous les angles possibles les problèmes posés par le lac Trummen, de tirer profit des extraordinaires possibilités de recherches que comporte cette tentative de régénération, et de faire de ce projet une opération pilote, une coopération entre les diverses disciplines écologiques et un groupe de travail technico-écologique a été mise en place.

Quelque vingt écologistes travaillent sur le projet depuis mars 68. L'essai de régénération a débuté en juillet 1970. Au cours de l'année $1970,0,5 \mathrm{~m}$ de boue ont été évacués par pompage, et en $19710,5 \mathrm{~m}$ seront également évacués; de sorte que le volume total des boues enlevées s'élèvera à $600000 \mathrm{~m}^{3}$. De plus, il y a les eaux chargées en boue qui représentent $50 \%$ environ du volume des boues proprement dites.

Au cours de la période précédant celle de la pollution, le rythme de croissance des sédiments au centre du lac Trummen était de $0,4 \mathrm{~mm}$ par an. Durant la période de réception des eaux usées, ce chiffre atteignit un maximum de $8 \mathrm{~mm}$ par an.
Les sédiments inaltérables, bruns et bien agglomérés du lac Trummen sont recouverts d'une «boue d'épandage » noire et sans consistance contenant entre autre choses, un fort pourcentage de phosphore, de plomb, de zinc et de cuivre. Dans la boue noire se produit un dégagement gazeux avec libération en milieu anaérobie d'une quantité considérable de phosphore et d'azote.

Le $\mathrm{pH}$ de l'eau était de 6 à 7 pendant l'hiver, alors qu'en été des valeurs atteignant 10 ont été fréquemment enregistrées; le chiffre le plus haut, mesuré au centre du lac, s'élève à 10,6 . Les teneurs en phosphate et en phosphore totales constatées dans le lac durant l'été étaient de l'ordre de 200 à $600 \mathrm{mg} / \mathrm{l}$, ce qui est 50 fois plus élevé que dans les lacs non pollués de la région.

Pendant l'été, les algues bleu-vertes étaient le composant principal du phytoplancton et leur génération était concentrée sur une couche trophogénique de 30 à $40 \mathrm{~cm}$ d'épaisseur. A proximité de la surface la production atteignit un maximum de $10 \mathrm{gC} / \mathrm{m}^{3}$ par jour, la production moyenne d'été s'élevait à $2,5 \mathrm{gC} / \mathrm{m}^{2}$ par jour et la production annuelle à $390 \mathrm{gC} / \mathrm{m}^{2}$ de surface du lac.

La turbidité prononcée de l'eau avait empêché la végétation de se développer en profondeur. La poussée des nénuphars et autres plantes émergentes s'était par contre bien développée en quantité.

Des essais de pompage des sédiments furent effectués, partie en mars et partie en août 1969. Dans le second cas $5000 \mathrm{~m}^{3}$ furent pompés, en liaison avec des essais de différents types de buses d'admission des pompes. La boue pompée a été utilisée pour des études sur la vitesse de décantation et d'assèchement, la réaction au gel et la composition de l'eau rejetée. Des essais ont été également effectués en vue de diminuer le pourcentage des nutrients, et de pouvoir se servir de la boue comme engrais.

Les sédiments pompés contenaient en moyenne 96 à $97 \%$ d'eau. Après $24 \mathrm{~h}$, la décantation se produisait. A une teneur de $87 / 88 \%$ un réseau de fissures rapprochées apparâtt dans la partie émergée des sédiments. Pendant l'été de 1970, la boue fissurée du bassin de décantation se couvrit d'une floraison uniforme de roseaux communs (typha-latifolia). Les fissures rapprochées et profondes ainsi que la prolifération des roseaux accélèrent l'assèchement.

Le procédé couramment utilisé pour la régénération du lac Trummen implique le pompage de 1 mètre de boue, opération qui est effectuée en deux étapes. La partie superficielle des terrains autrefois cultivés en bordure du lac a été décapée. A l'aide de bulldozers, une série de bassins de décantation ont été creusés dans la moraine primaire sous jacente et la boue a été déversée dans ces bassins. Après décantation de la boue, l'eau est renvoyée au lac après être passée par une station de précipitation au sulphate d'alumine. L'eau ainsi traitée est claire et a une teneur totale en phosphore de $30 \mathrm{mg} / \mathrm{l}$.

Les différences mises en évidence par essai de croissance des algues sont considérables entre eaux de rejet non traitées et traitées, lesquelles constituent un mélange d'eau de lac et d'eau interstitielle.

Après assèchement, et de préférence congélation, les boues de pompage peuvent être utilisées comme engrais. L'emplacement servant actuellement de bassin de décantation sera probablement utilisé ultérieurement comme terrain de jeu.

Après enlèvement de $0,5 \mathrm{~m}$ de sédiments en 1970 , le lac Trummen s'est nettement écarté au point de vue limnologique des conditions observées au cours des années antérieures. Par suite de l'augmentation de la transparence, la distribution verticale de la génération primaire de phyto- 
plancton a également augmenté, et on a observé un changement dans la composition des espèces.

Le pourcentage de phosphore total de la couche aquatique superficielle avait diminué de $50 \%$ en 1970 , et dans le courant de l'hiver 1970-71, de très bonnes conditions d'oxygénation ont été constatées par rapport aux hivers précédents, tous caractérisés par une déficience d'oxygène totale ou très importante et la mortalité des poissons.

Le but de la régénération du lac Trummen et des autres lacs, considérés comme projets pilotes, est précisément de faire fructifier la structure et la fonction limnologique du système écologique.

Sans une telle connaissance, la régénération des lacs ne peut être réalisée.

\section{Lac Järlasjön et lac Växjösjön}

Tandis que le lac Trummen est un lac peu profond caractérisé par une circulation totale de l'eau pendant l'été, le lac Järlasjön et le lac Växjösjön présentent une stratification calorifique, permanente pour le premier, quasiment permanente pour le deuxième.

\section{Lac Järlasjön}

Sa profondeur est de $23 \mathrm{~m}$, sa superficie approximative de $1 \mathrm{~km}^{2}$, et son volume de $7,5 \mathrm{Mm}^{3}$. La stratification calorifique est très tranchée: en juillet: la thermocline se situe à une profondeur de 7 à $8 \mathrm{~m}$.

Le lac a été utilisé comme réceptacle d'égouts et d'eaux usées industrielles. Depuis lors, on a constaté une complète insuffisance d'oxygène dans la plus grande partie des couches profondes durant l'été, et pendant l'hiver, les conditions de l'oxygénation ont été également très mauvaises.

Evidemment, les conditions anaérobies eurent comme résultat une sédimentation superficielle réduite, et un enrichissement considérable du phosphate et de l'ammonium dans les couches inférieures. Au-dessous d'environ $8 \mathrm{~m}$, il n'y avait pratiquement plus aucune vie animale, et les poissons s'entassaient dans les couches aquatiques supérieures.

Il n'y a aucun avantage à retirer du mélange de la grande quantité d'eaux des couches inférieures pauvres en oxygène avec celles des couches supérieures riches en oxygène, puisque cela peut provoquer la désoxygénation de toute la masse de l'eau.

Dans le cadre de l'expérience du lac Järlasjön une pompe actionnée par l'air comprimé, spécialement conçue, fut utilisée pour transporter l'eau des couches inférieures vers un bassin installé en surface. La pompe consiste simplement en un tube avec une admission d'air, concentrique à sa partie centrale inférieure. Quand l'air est admis, les bulles montent dans le tube, créant une différence de pression qui entraîne l'eau.

A la partie supérieure du tube, l'eau est déversée dans un bassin en plastique, où elle reçoit si nécessaire un supplément d'aération. L'eau est immédiatement envoyée dans les couches inférieures à l'aide de tuyaux en plastique, sans augmentation de la température, puisque ceci pourrait perturber la stratification calorifique du lac. Par suite du contact étroit de l'air et de l'eau dans le tuyau de pompage, la teneur en oxygène de l'eau peut être ramenée à un niveau situé entre 70 et $90 \%$ de la saturation. Les tuyaux assurant le retour de l'eau sont dispersés sur une surface aussi grande que possible dans les couches infé-
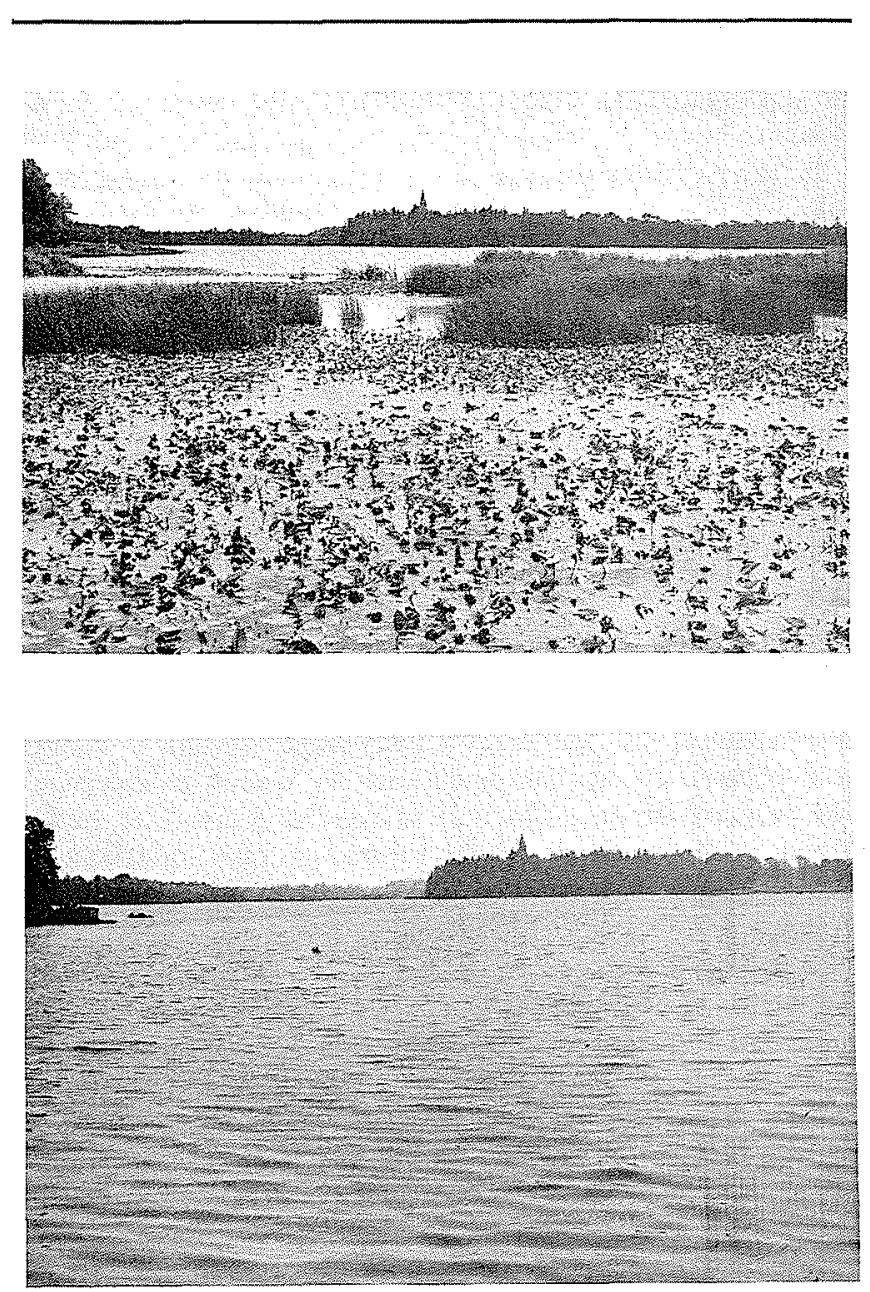

2/Aspects du lac Trummen en 1964 (cliché du haut) et en 1971, c'est-à-dire avant et après l'assainissement.

Lake Trummen in 1964 (above) and 1971, i.e. before and after the restoration.

(Photos S. Björk)

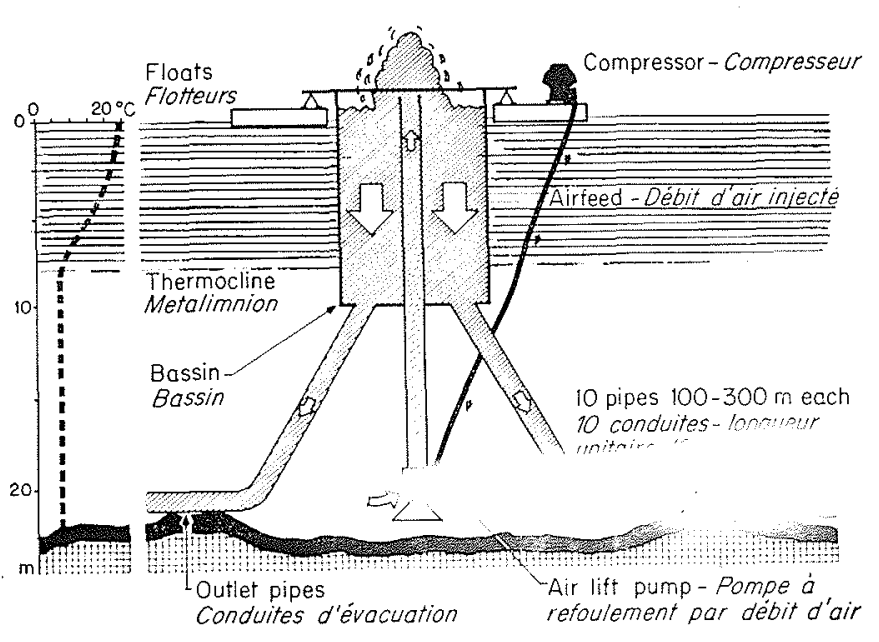

3/Dispositions techniques adoptées au cours de l'expérience effectuée dans le lac Järla en vue de l'aération de l'hypolimnion sans perturbation de la stratification thermique. (Cliché fourni par les Laboratoires Centraux de la société Atlas Copco).

The technical arangements when aerating hypolimnion water in the Lake Järla experiment without disturbing the thermal stratification. (From Atlas Copco Central Laboratories.) 


\section{S. BJÖRK}

rieures, afin d'obtenir un équilibre aussi parfait que possible de la teneur en oxygène. Ceci a également pour but d'empêcher la diffusion des nutrients contenus dans les sédiments.

La partie limnologique du programme de recherches comporte des études sur l'eau, les sédiments, le plancton, la faune des fonds et les poissons, ceci avant, pendant et après les essais d'aération, tandis que la partie technique comprend l'étude du transfert des masses à l'intérieur du lac, et l'enregistrement des renseignements météorologiques.

Depuis la fin mai 1970 , date à laquelle la stratification avec manque total d'oxygène dans les fonds fut mis en évidence jusqu'à la fin octobre 1970 - quand redémarra la circulation totale d'automne - les couches inférieures du lac Järlasjön furent aérées. Pour cet essai, le débit de la pompe était de $32 \mathrm{~m}^{3} / \mathrm{mn}$.

Le but de l'essai d'aération de 1970 était de changer le milieu anaérobie des couches inférieures en milieu aérobie sans perturber la stratification calorifique. Avañ le début de la période de stagnation de l'été, la teneur en oxygène à la profondeur de $21 \mathrm{~m}$ était d'environ $8 \mathrm{mg} / 1$, et nulle part dans le lac le degré de saturation était inférieur à $60 \%$. La valeur moyenne pour les couches inférieures était de $7 \mathrm{mgO}^{2} / 1$.

En même temps que la teneur en oxygène des couches inférieures de l'eau augmentait, la concentration en ammonium et en phosphore diminuait. Par comparaison avec les conditions du dernier été de 1969 , les concentrations à la période correspondante de 1970 , ont diminué de 800 à $50 \mathrm{mg} \mathrm{NH} \mathrm{NH}^{3}-\mathrm{N}$ par $\mathrm{m}^{3}$, et de 950 à $50 \mathrm{mg} \mathrm{PO}^{4}-\mathrm{P}$ par $\mathrm{m}^{3}$. La teneur dans les eaux intersticielles des sédiments de surface est d'environ $2,5 \mathrm{mg} / 1$. En laboratoire, des essais concernant l'échange de phosphore, ont montré que, entre les sédiments et l'eau, la teneur en phosphore de l'eau était d'environ $1 \mathrm{mg} / \mathrm{l}\left(\mathrm{PO}^{4}-\mathrm{P}\right)$ en milieu anaérobie, c'est-àdire, environ les mêmes que celles constatées dans le lac non-traité. Les sédiments sont étudiés par exemple sous l'angle de leur capacité d'absorption de l'oxygène. La production de matières organiques par les algues est mesurée à l'aide de la technique du $\mathrm{C}^{\mathbf{1 1}}$. Le plancton est étudié en ce qui concerne son changement de volume et de composition spécifique par suite du traitement de régénération. Des échantillons de la faune des fonds sont examinés en vue de déterminer les changements de composition des espèces. Un intérêt spécial s'attache à déterminer jusqu'à quel point de nouvelles zones en profondeur peuvent être peuplées par des espèces animales par suite de l'amélioration des conditions d'oxygénation dans cette zone.

\section{Lac Växjösjön.}

Profondeur $6,5 \mathrm{~m}$, superficie approximative $1 \mathrm{~km}^{2}$, volume $3,3 \mathrm{Mm}^{3}$.

Le lac présente presque toujours une stratification calorifique pendant l'été. Cependant des vents violents peuvent mélanger les eaux, et rendent le lac d'égale température.

On a déversé dans le lac, des eaux d'égouts et des eaux usées, d'origine industrielle. Bien qu'aucune eau usée n'ait été rejetée dans le lac depuis de longues années, les symptômes correspondent à ceux d'un bassin dont les capacités d'absorption ont été dépassées et ont continué à se manifester. Au cours de l'été, il y eut jusqu'en 1969 une vigoureuse floraison d'algues bleu-vertes, et durant la période de stagnation, les conditions de fermentation anaérobie apparurent dans les couches voisines du fond, et la teneur en $\mathrm{PO}^{4}-\mathrm{P}$ atteignit $900 \mathrm{mg} / \mathrm{m}^{3}$ dans les couches inférieures de ce lac, à l'origine du type oligotrophe.

Comme, dans le lac Växjösjön, le volume des couches inférieures, par rapport au volume total de l'eau, est faible, il n'y a pas d'inconvénient à mélanger ces différentes couches. Le principe de la régénération est par conséquent d'éviter la formation d'une stratification au sein des couches inférieures. On y parvient en insufflant de l'air à l'aide de flexibles percés de trous, qui sont ancrés au fond du lac. Les bulles d'air ascendantes provoquent un brassage général du lac, et la masse entière de l'eau est en tout temps en excellent contact avec l'atmosphère. Dans le même temps, ce brassage, ajouté à la diffusion des bulles d'air, assure une pénétration de l'oxygène dans l'eau.

A l'aide de l'équipement technique mis en curre au lac Växjösjön, il est possible de rendre la masse du lac d'égale température, et d'arriver à de bonnes conditions d'oxygénation de la surface au fond, ceci en deux jours. L'élaboration des nutrients est évidemment fortement diminuée, par suite du passage de milieu réducteur en milieu oxydant à la surface des sédiments.

Le programme des études des changements limnologiques résultant de l'aération du lac Växjösjön est le même que celui du lac Järlasjön. Notamment les recherches sur la prolifération des espèces animales dans les zones les plus profondes sont du plus haut intérêt.

\section{Lac Hornbörgas.}

On a abaissé le niveau du lac Hornbörgas à cinq reprises de 1802 à 1933. Les deux derniers abaissements, effectués essentiellement en 1911 et en 1933, ont eu pour résultat la prolifération presque générale d'une luxuriante végétation de surface.

Avant l'abaissement de son niveau, la profondeur du lac était de $3 \mathrm{~m}$ à l'étiage normal d'été, et sa superficie s'élevait à $30 \mathrm{~km}^{2}$. A l'intérieur d'une faible partie endiguée, la profondeur estivale maximale est de $80 \mathrm{~cm}$. Pendant l'année 1953-1954, une superficie de $25 \mathrm{~km}$ ² était entièrement à sec pendant l'été. Depuis 1954 on a divisé le lac en une partie endiguée restant partiellement en eau, et une partie extérieure à sec pendant l'été.

Les abaissements du niveau avaient été effectués en vue de gagner des terrains cultivables. Depuis 1933, le lac a été complètement endigué, mais bien que les eaux affluentes soient envoyées directement au déversoir, il n'a pas été possible de cultiver la surface du lac.

Depuis que l'augmentation du rythme de croissance des sédiments dans la partie du lac court-circuitée du point de vue hydraulique est très prononcée et que les terrains environnants drainés s'enfoncent, un processus de nivellement se déclare, qui devient de plus en plus perceptible. Le lac Hornbörgas a toujours été caractérisé par des variations sensibles de son niveau d'eau - le bassin de réception est de $616 \mathrm{~km}^{2}$, alors que la surface du lac, comme indiqué plus haut, est de $25 \mathrm{~km}^{2}$ - et par conséquent l'accroissement de l'apport des sédiments et l'affaissement des terrains environnants augmentera sérieusement le risque des inondations.

Le lac Hornbörgas a été considéré comme l'un des plus importants d'Europe, en ce qui concerne les oiseaux aquatiques, et sa valeur, aussi bien pour le séjour que pour le nichage des oiseaux a été soulignée. Les nouvelles conditions limnologiques résultant de l'abaissement du niveau. ont eu pour résultat une rapide diminution de la valeur du lac au point de vue ornithologique. Au point de vue économique, l'abaissement du niveau du lac a causé de grosses pertes. Si lon considère en autre choses les prévi- 
sions d'affaissements signalées plus haut, on peut s'attendre à des pertes encore plus considérables, au point de vue économique.

Le gouvernement suédois a fait une étude de la valeur du lac Hornbörgas, du point de vue de la protection de la nature. On en conclut que « la régénération du lac Hornbörgas est l'un des projets les plus urgents dans ce pays, du moins en ce qui concerne la protection de la nature dans ce domaine particulier $\gg$.

L'office national de la Protection de la Nature a reçu du gouvernement l'ordre d'étudier la question de l'avenir du lac Hornbörgas, et son bureau de protection de la nature dirige le groupe d'enquêteurs, dont le premier travail fut, fin 1967, de décider si le lac pourrait être régénéré suivant les buts qu'on se proposait, c'est-à-dire de remodeler le lac de telle façon qu'il puisse retrouver en permanence son rôle de lac pour les oiseaux aquatiques.

Parmi les groupes qui effectuent l'enquête, on peut citer : les hydrotechniciens, agriculteurs, biologistes et ornithologues.

Le résultat de plus d'une année d'étude fut une réponse nettement affirmative de principe sur la question de savoir, s'il était possible de régénérer le lac Hornbörgas. La partie restante de la période de recherche jusqu'à fin 1970, fut consacrée à la mise au point des méthodes et des programmes de détail, pour une régénération définitive, en même temps qu'une évaluation du coût de l'opération. On a prévu que l'élévation du niveau de l'eau serait de 1 mètre au moins au dessus de l'étiage normal d'été.

Avec la collaboration de l'office national pour la Protection de la Nature, de l'Office Royal du Marché du Travail, de l'Office des Forêts et de la Société de Chasse Locale, un programme expérimental aux dimensions réelles fut élaboré. Le but était alors de mettre en œuvre des méthodes permettant de résoudre de façon économiquement favorable les problèmes écologiques qui se posaient. Ceci a été effectué grâce à une collaboration encourageante et fructueuse de la limnologie, l'ornithologie et de la limnotechnologie.

Avant d'entreprendre un réhaussement définitif du niveau de l'eau, il faut effectuer un travail préliminaire considérable Ainsi il est nécessaire d'être bien sûr qu'un fort courant continuera à traverser le lac de façon continue, que les conditions pour le mouvement des glaces sont réunies, que les masses de gros détritus accumulés seront résorbées, et que le développement quantitatif et qualitatif de la végétation sera repensé.

Dans ce dernier cas, la question primordiale est celle
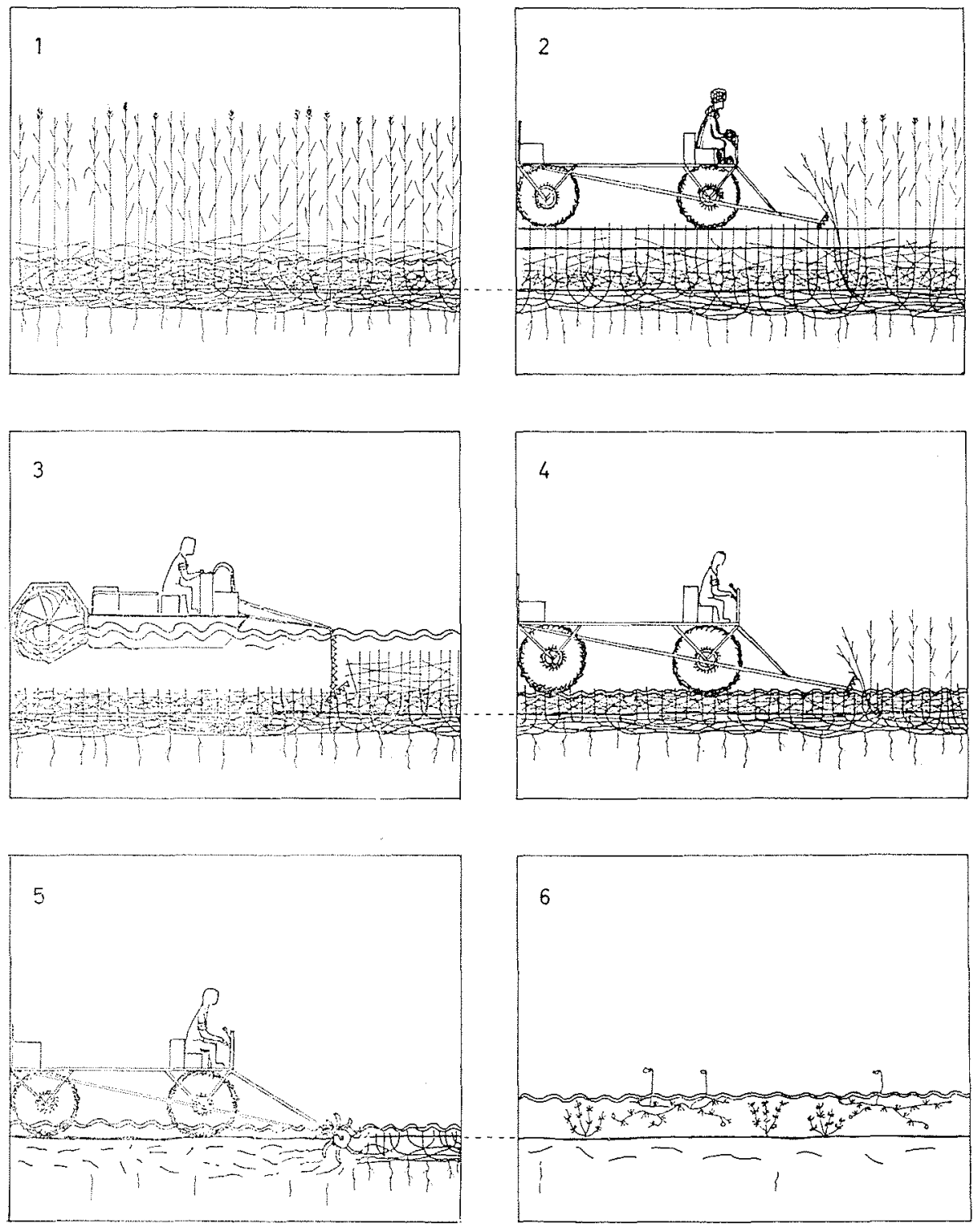

4/Schéma envisagé en vue de l'assainissement du lac Hornborga, établi sur la base des résultats des expériences effectuées sur le terrain en 1968-1970. 1. Aspect du terrain non-aménagé : couverture végétale constituée entièrement de roseaux, limon consolidé recouvert d'un « tapis» de gros débris végétaux, et présence d'un enchevêtrement très dense de racines dans la couche supérieure du limon / 2. Premiers travaux : coupe des roseaux en hiver (à raison de $2 \mathrm{ha} / \mathrm{h}$ ) la paille coupée étant brûlée / 3. A l'époque des crues printanières, déchaumage et élimination des tiges de roseau couchées sur le fond à l'aide d'une faucardeuse sur pontons / 4. Lors de l'étiage estival, coupe des pousses, et préparation du sol à l'aide de roues spéciales sur l'engin de coupe. / 5. Labourage définitif du fond à l'aide d'un « rotoculteur 》/ 6. Remplacement de la végétation émergente par des espèces sub-aquatiques, et implantation d'une faune de fond d'une très grande diversité d'espèces et très abondante.

Scheme to be practiced in a restoration of Lake Hornborga. Results from the field experiments 1968-1970.

I. Initial state: The area overgrown by common reed, the consolidated mud covered by coarse detritus and a dense root felt developed in the top layer of the mud / 2. The work starts with cutting during the winter. Capacity 2 hectars per hour. The reed material is being burnt / 3. During the spring high water period pontoon-equipped mowing machines are used for reducing the stubble and clearing the bottom from the layer of horizontal reed stems / 4. At low water in the summer the green shoots are cut and at the same time it is possible to prepare the bottom by means of special wheels / 5. Final preparation of the bottom by a rotor cultivator / 6. The emergent vegetation is replaced by submerged plants, and bottom fauna communities rich in species and individuals are developed. 


\section{S. BJÖRK}

de la réalisation d'une redistribution de l'élaboration de la végétation émergée en une végétation submergée. Au début de l'étude, la végétation émergée se composait de zones peuplées principalement de roseaux ordinaires $\left(12 \mathrm{~km}^{2}\right)$ et de joncs avec des espaces comportant également des saules en buissons.

L'étude a démontré qu'il était toujours possible de régénérer le lac dans sa moitié nord, et sur une surface de $10 \mathrm{~km}^{2}$ d'eau libre. La zone riveraine peut retrouver là, dans une grande mesure, les caractéristiques d'un lac peu profond exposé au vent, où des rives minéralisées et des biotopes convenant aux échassiers peuvent être créés. Les sédiments de la végétation primitive de la partie nord du lac sont des boues calcaires. Dans la partie sud, et de même dans les autres zones non exposées, les sédiments sont des boues de détritus. Le fond est abondamment recouvert de joncs.

Peu de temps après le réhaussement du niveau de l'eau, les racines agglomérées flotteront et croîtront à nouveau.

Afin de rendre attrayantes pour les oiseaux ces régions auxquelles l'abaissement du plan d'eau a causé les plus graves dommages, et qui sont extrêmement monotones, une mosaïque d'eau libre et de refuges (biotopes) pour le nichage peuvent être créés.

L'envahissement des plantes immergées dans les zones aquatiques creusées par des excavatrices amphibies se fait très rapidement. La croissance de la végétation immergée s'est effectuée également de façon rapide dans les zones expérimentales où les champs denses et homogènes de roseaux communs ont été nettoyés à l'aide de moyens mécaniques (faucheuses amphibies montées sur pontons) et où le fond a été traité en même temps de telle façon que la boue colmatée peut être mise à l'air.

Au stade actuel de l'étude, un nettoyage complet et un traitement du fond des zones recouvertes de roseaux exige 8 à $10 \mathrm{~h}$ de machine par hectare. En comparaison, la culture des céréales en Suède, exige 10 à $21 \mathrm{~h}$ de machine par hectare de terre cultivable. L'attention doit être attirée sur le fait que l'opération entreprise au lac Hornbörgas n'est pas susceptible de se renouveler

Afin d'étudier la possibilité de transport des matériaux sur l'eau et sur la glace, un large dégagement a été opéré dans la direction longitudinale du lac, débouchant sur les parties exposées du rivage. On a ainsi démontré qu'il était possible d'instaurer le transport d'énormes quantités de débris grossiers vers le rivage, où on pourrait par la suite les brûler, afin de réduire leur volume.

Ces mesures d'ordre limnologiques ont eu des résultats très positifs sur la venue des oiseaux. Ces résultats ont été régulièrement enregistrés par le groupe de travail ornithologique.

Discussion

M. le Président remercie vivement le conférencier et son traducteur et avant d'ouvrir la discussion fait part, comme suit, de ses observations personnelles

Au cours de ma carrière, j'ai été surtout intéressé par les lacs un peu profonds; pour moi, un lac est une étendue d'eau ayan au moins $20 \mathrm{~m}$ de profondeur. C'est le cas des lacs de l'Ain, du Jura et de la Savoie. J'ai donc été particulièrement intéressé par ce qui a été dit du Lac Järla, profond de $23 \mathrm{~m}$.

C'est évidemment un petit lac puisqu'il a un killomètre carré de superficie et une capacité de 5 millions de mètres cubes. Mais je pense que le procédé utilisé est très efficace et intéressant a priort.

On a essayé, il y a quelques années, en Allemagne, en Pologne et en Suisse aussi, d'envoyer de l'oxygène ou de l'air comprimé dans le fond des lacs pour réaliser un brassage des eaux; mais comme l'a très bien souligné le Professeur Brork, il est essentiel de ne pas mélanger les eaux épilimniques et les eaux hypolimniques pour no pas rompre la stratification thermique et augmenter la température profonde. C'est ce qu'on fit en Allemagne fédérale, dans le lac de la Wahnbachtalsperre en créant par air comprimé un courant d'eau dans le seul hypolinnion par utilisation d'un tube vertical de $2 \mathrm{~m}$ de diamètre et de $40 \mathrm{~m}$ de long percé de trous à une dizaine de mètres de la surface.

A l'ouverture de la discussion, M. SkLenard pose la question suivante: le lac de Nantua est, me dit-on, un « lac-pilote » pour la réoxygénation. Est-il prévu d'y appliquer un procédé analogue à celui utilisé au lac Järla?

Personnellement, répond $M$. le Président, je ne suis pas tellement partisan d'utiliser le lac de Nantua comme « lac-pilote ». C'est, en effet, un lac qui a $2,5 \mathrm{~km}$ de long, $600 \mathrm{~m}$ de large et contient 40 millions de mètres cubes d'eau. C'est donc un lac nettement plus grand que le lac Järla avec son kilomètre carré de superficie et ses 5 millions de mètres cubes d'eau. Mais je dois dire qu'après la conférence de M. BJORK et la description de son système mobile, je serais peut être moins hostile que je l'étais auparavant à une telle expérience. Mais je ne connais pas le programme envisagé actuellement par les Services responsables.

Il faut dire qu'en Pologne, en Suisse et dans beaucoup d'essais faits en Allemagne, les résultats ne semblent pas extrêmement brillants sur des lacs qui ont une certaine profondeur; dans les autres lacs, le problème est tout à fait différent.
J'ai visité moi-même en Pologne le petit lac de Kortowo $\left(1 \mathrm{~km}^{2}\right)$ il y a quelques années. J'ai rencontré le Professeur Orszewski qui avait été chargé de l'opération (siphonage des eaux de fond); il avait considéré que les résultats étaient assez décevants. Depuis, il est vrai, il a changé d'avis.

En Suisse, dans le lac de Pfäffikon, l'ingénieur Murler a utilise un système un peu analogue à celui qui nous a été présenté aujourd'hui. Il envoyait de l'air comprimé dans un grand tube de $2 \mathrm{~m}$ de diamètre environ pour amener en surface l'eau du fond qui s'y oxygénisait. Il a obtenu un certain résultat, mais peu important, eu égard aux dépenses engagées.

Néanmoins, je partage l'opinion autorisée du Professeur JAAG, président de la Fédération européenne de protection des eaux, et reste un peu comme lui sceptique sur l'efficacité réelle de ces procédés, mais bien entendu, je trouve que cela peut être très intéressant d'essayer un système flottant comme celui du Professeur Brork. Ce qui me fait un peu hésiter dans le cas du lac de Nantua c'est qu'il s'agit là d'un lac déjà assez grand.

M. Sterling demande au Professeur BJork s'il pourrait comparer brièvement les résultats obtenus et le coût des opérations pour les deux lacs peu profonds - dont il a parlé - traités l'un par dragage et l'autre par insufflation d'air.

Traduit par M. LaRRé, M. le Professeur BJork répond en anglais.

Je n'ai pas encore beaucoup d'indications sur les opérations d'aération car elles sont très récentes.

En ce qui concerne les frais engagés dans le cas du premier lac, celui qui a été traité par succion, la dépense limitée aux « interventions techniques », a été d'environ 2 millions de couronnes ( $2 \mathrm{~F}$ au $\mathrm{m}^{2}$ ). Pour une restauration finale et totale, il faudrait compter beaucoup plus. Il faut dire qu'en Suède, on essaie de lutter. seulement contre la pollution \& historique " des lacs: on a enlevé la couche historique de boue, et à partir de là, on estime que l'opération est terminée et que désormais le lac évoluera bien.

En ce qui concerne le deuxième lac, il faudrait compter environ 10 centimes au mètre carré.

L'un des principaux résultats des expériences a été la formation d'écologistes qui maintenant sont capables de travailler avec des ingénieurs. En Suède, comme dans bien d'autres pays, nous avons beaucoup d'ingénieurs, mais peu d'écologistes.

M. THIRrIot pose la question suivante: 
Pourrait-on avoir une idée du rendement de l'injection d'air et savoir, par exemple, ce qui est le plus important de l'insufflation d'air comprimé à la base du tuyau qui entrâne l'eau, ou de l'aération provoquée dans la boursouflure superficielle due à l'agitation turbulente au sommet du tuyau?

M. BJORK indique, qu'en pratique, on utilise une pompe à êmulsion du type Mamouth, dont le rendement en pompage est faible mais à la sortie de cet appareil, la teneur en oxygène dissous dans l'eau est de l'ordre de $80 \%$ de la concentration de saturation. C'est probablement dans l'intumescence superficielle produite par la pompe que se réalise la majeure partie du processus d'oxygénation.

M. Remenieras rappelle l'intérêt que pourrait éventuellement pré- senter pour ce genre d'application l'emploi de compresseurs d'air hydrauliques à émulsion (*) installés sur les affuents du lac; l'eau ayant traversé ces appareils est, en outre, toujours saturée d'oxygène dissous et contient une multitude de très fines bulles d'air suroxygéné qui lui donnent une certaine opalescence.

M. le Président clôt la discussion et donne la parole à M. BaletTe qui, en l'absence de l'auteur en mission à l'étranger, expose la communication de M. GoMeLla. (*) G. Rearenieras, Les compresseurs d'air liydraulique à émulsion.
Mémoires et travant. de la Société Hydrotechtique de France, supplément au volume I (1961)

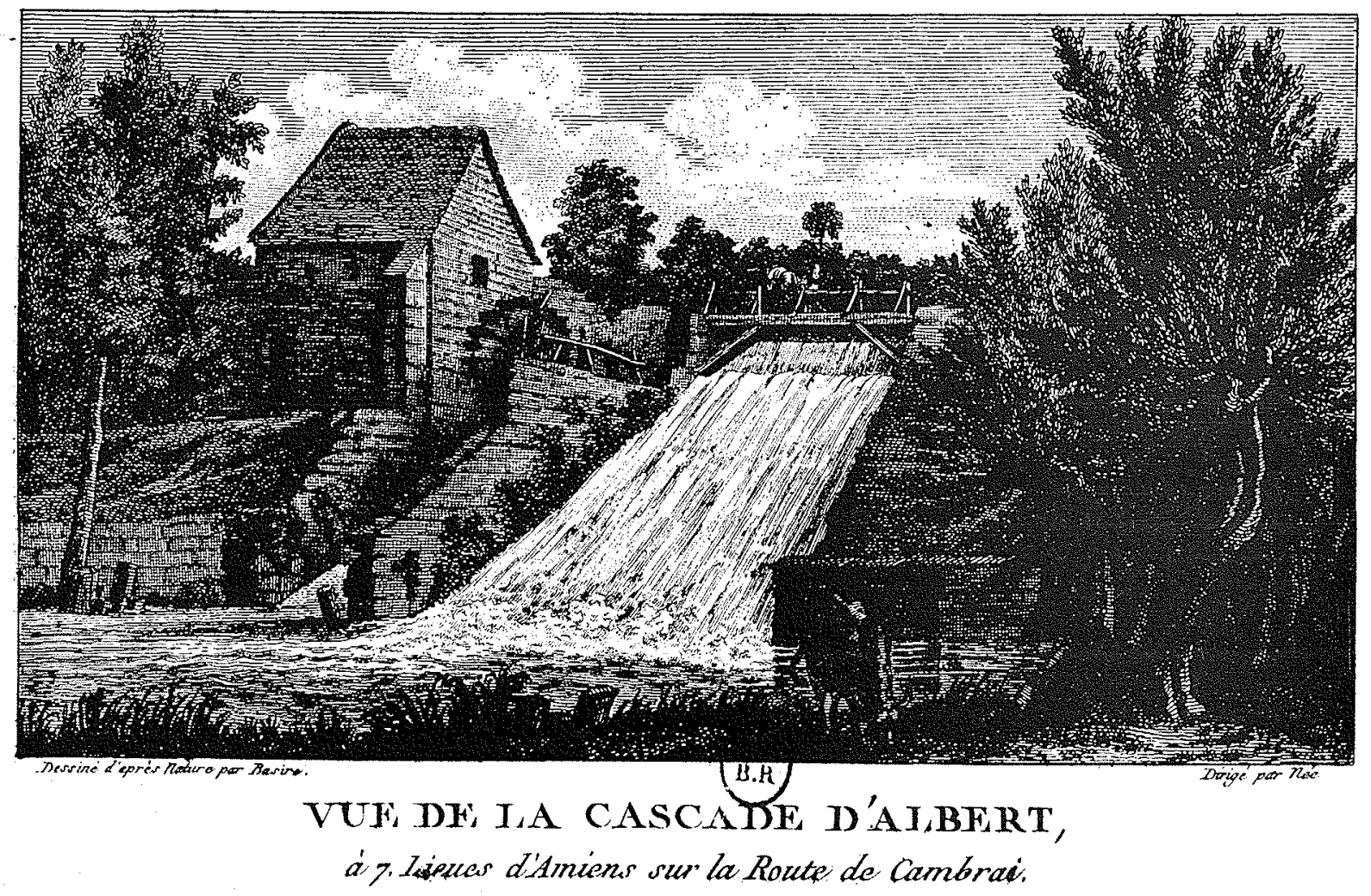

Picantie. $n^{\circ} 8$ 


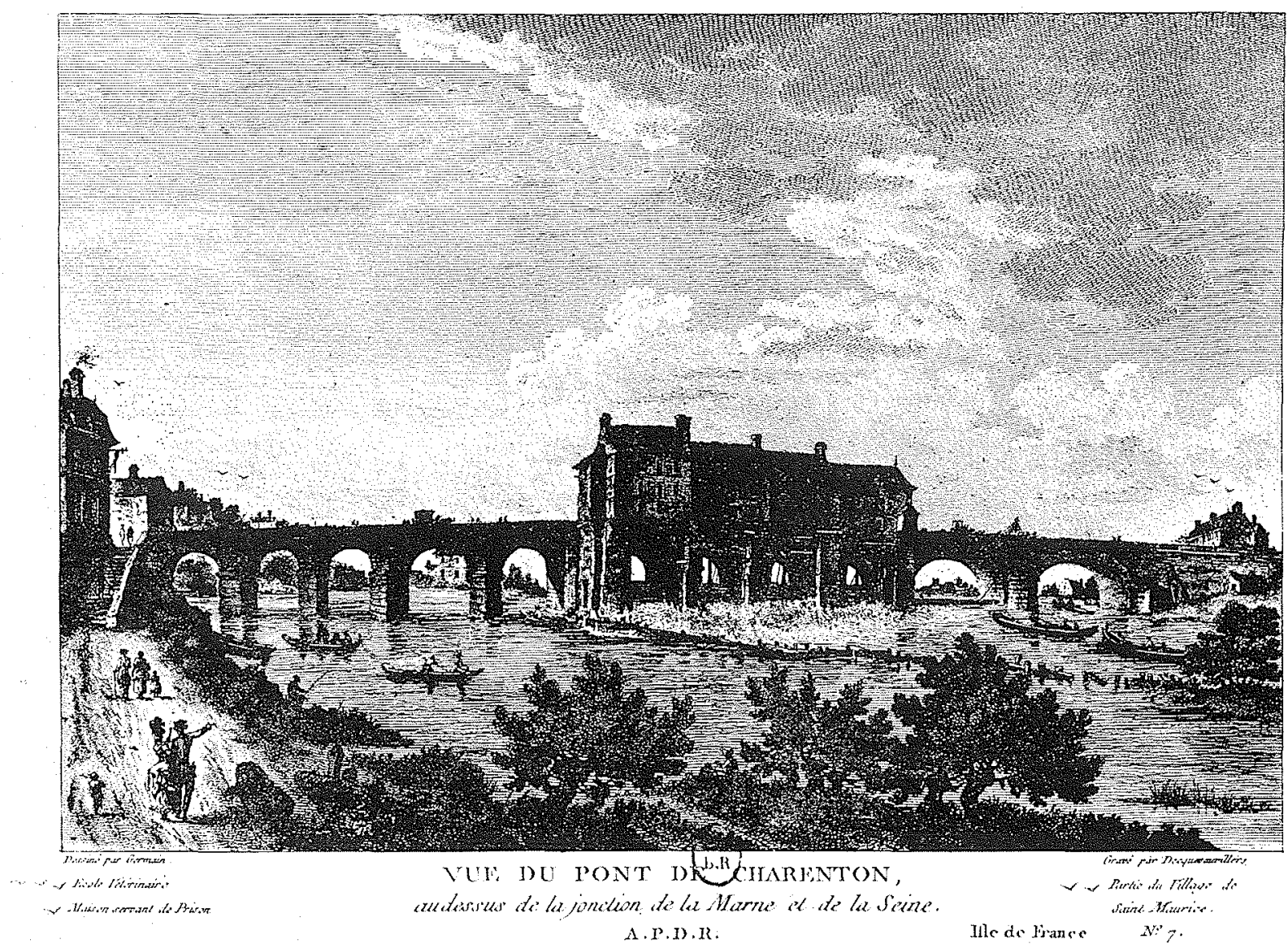

